Consideration of whether to extend immunisation against hepatitis B to infants of all mothers positive for hepatitis B surface antigen will need to take into account the resources of the hospital or the district in terms of the fivefold increase in workload which it is estimated that this would entail. In any event, differentiation of carriers by tests for hepatitis $\mathrm{B} \mathrm{e}$ antigen and antibody should be continued as priority should always be given to infants at high risk of persistently carrying hepatitis B surface antigen and later developing cirrhosis and primary hepatic carcinoma.

In conclusion, the programme has had one disappointing aspect, which is that so far consultants in only half of the hospitals with obstetric departments have collaborated. Hepatitis B vaccine is now readily available, stocks of specific hepatitis B immunoglobulin for immunisation are assured, and we hope that our successful results will encourage all those concerned to take part in future.

We thank all the members of the obstetric, paediatric, and microbiological teams who collaborated; and Mrs Valerie Little, Hepatitis Epidemiology Unit, who acted as assistant coordinator for the study and organised the computer analysis.

1 Beasley RP, Lin C-C, Hwang L-Y, Chien C-S. Hepatocellular carcinoma and hepatitis B virus: a prospective study of $22707 \mathrm{men}$ in Taiwan. Lancet 1981;ii:1129-33.
2 Sung J-L, Chen D-S. Maternal transmission of hepatitis B surface antigen in patients with hepatocellular carcinoma in Taiwan. Scand $\mathcal{f}$ Gastroentero 1980;15:321-4

3 Beasley RP, Trepo C, Stevens CE, Szmuness W. The e antigen and vertical transmission of hepatitis B surface antigen. Am $\mathcal{F}$ Epidemiol 1977; 105:94-8.

4 Reesink HW, Reerink-Brongers EE, Lafeber-Schut BJT, KalshovenBenschop J, Brummelhuis HGJ. Prevention of chronic HBsAg carrier state in infants of $\mathrm{HBs} A \mathrm{~g}$-positive mothers by hepatitis $\mathrm{B}$ immunoglobulin. Lancet 1979;ii:436-8.

5 Beasley RP, Hwang L-Y, Lin C-C, et al. Hepatitis B immune globulin (HBIG) efficacy in the interruption of perinatal transmission of hepatitis B virus carrier state. Initial report of a randomised double-blind placebo-controlled trial. Lancet 1981; ;ii:388-93.

6 Beasley RP, Hwang L-Y, Lee GC-Y, et al. Prevention of perinatally transmitted hepatitis B virus infections with hepatitis B immune globulin and hepatitis B vaccine. Lancet 1983;ii:1099-102.

7 Polakoff S. Immunisation of infants at high risk of hepatitis B. Br Med $\mathcal{J}$ 1982;285: 1294-5.

8 Polakoff S. Immunizing infants at high risk of hepatitis B. Health Visitor 1982;55:668.

9 Polakoff S. Transmission from mother to infant of hepatitis B virus infection Midwives Chronicle and Nursing Notes 1983 Jan:4-5.

10 Cameron CH, Combridge BS, Howell DR, Barbara JAJ. A sensitive immunoradiometric assay for the detection of hepatitis B surface antigen. 7 Virol Methods 1980;1:311-23.

11 Ferns RB, Tedder RS. Detection of both hepatitis Be antigen and antibody in a single assay using monoclonal reagents. F Virol Methods 1985;11:231-9.

12 Cohen BJ, Hewish RA, Mortimer PP. Comparison of radioimmunoassay and counter-immunoelectrophoresis for the detection of antibody to hepatitis B core antigen. $\mathcal{I}$ Virol Methods 1981;2:181-92.

13 Beasley RP, Stevens CE. Vertical transmission of $\mathrm{HBV}$ and interruption with globulin. In: Vyas GN, Cohen SN, Schmid R, eds. Viral hepatitis. Philadelphia: Franklin Institute Press, 1978:333-4

14 Beasley RP, Hwang L-Y, Stevens CE, et al. Efficacy of hepatitis B immun globulin for prevention of perinatal transmission of the hepatitis B virus carrier state: final report of a randomized double-blind placebo-controlled trial. Hepatology 1983;3:135-41.

15 Polakoff S. Immunoprophylaxis of infants born to hepatitis B virus exposed mothers. Arch Dis Child 1986;61:1242-3.

(Accepted 7 April 1988)

\title{
Clinical comparison of extracorporeal shock wave lithotripsy and percutaneous nephrolithotomy in treating renal calculi
}

Nicholas Mays, Sabri Challah, Swatee Patel, Edward Palfrey, Rosemary Creeser, Pragati Vadera, Peter Burney

\section{Abstract}

Study objective-To compare extracorporeal shock wave lithotripsy and percutaneous nephrolithotomy for efficacy in treating renal calculi.

Design-Non-randomised multicentre cohort study with 3 month follow up and 13 month data collection period.

Setting-Lithotripter centre in London, tertiary referral hospital, and urological clinics in several secondary and tertiary care centres.

Patients-933 of 1001 patients treated by lithotripsy at the lithotripter centre were compared with 195 treated by nephrolithotomy. Missing patients were due to incomplete collection of data. Age and sex distributions and characteristics of the stones were similar in the two treatment groups. Two patients died in the lithotripsy group. Three month follow up was achieved in about $84 \%$ of both groups (783/933 for lithotripsy; 163/195 for nephrolithotomy).

Interventions-The nephrolithotomy group had surgical nephrolithotomy alone. In the lithotripsy group $83 \%$ (774/933) had lithotripsy alone, 11\% (103/ 933) had combined lithotripsy and nephrolithotomy, and $6 \%(56 / 933)$ had lithotripsy plus ureteroscopy. Single and combined lithotripter treatments were analysed as one group and compared with nephrolithotomy.

End point-Presence of stones three months after treatment.

Measurements and main results-Presence of residual stones was assessed by plain radiography, ultrasonography, or intravenous urography. After adjustment for age and size and position of stone for patients with single stones the likelihood of being free of stones three months after treatment was significantly greater in the nephrolithotomy group than the lithotripsy group (odds ratio $6.6 ; 95 \%$ confidence interval 3.0 to 14.6 ) and the response was particularly pronounced with staghorn calculi $(62 \%$ $(8 / 13) v 15 \%(141 / 96)$ patients free of stones after nephrolithotomy and lithotripsy, respectively).

Other findings-19\% $(146 / 775)$ of patients who had had lithotripsy had to be readmitted within three months after treatment compared with $14 \%(23 / 162)$ who had nephrolithotomy; and 64\% (94/146) of readmissions after lithotripsy were for complications compared with $30 \%(7 / 23)$ of readmissions after nephrolithotomy.

Conclusions-Nephrolithotomy may be preferable to lithotripsy for treating renal stones and it may not be wise to invest heavily in lithotripsy facilities.

\section{Introduction}

Between 1985 and 1987 a single extracorporeal shock wave lithotripter was available to the NHS at St Thomas's Hospital, London. The Dornier machine at St Thomas's is an example of the first generation of the new technology of lithotripsy. It was joined in mid1987 by a second generation machine installed in Manchester by the North Western Regional Health Authority. Several other regional health authorities in England are currently considering the merits of purchasing their own second generation extracorporeal shock wave lithotripters for treating renal calculi. In these regions stones are currently removed by conventional open surgery or by percutaneous nephro- 
lithotomy, with limited access to the lithotripter in London. The purchase of further lithotripters by the NHS should be determined by the effectiveness, acceptability to patients, and relative cost of lithotripsy when compared with percutaneous nephrolithotomy or open surgery.

The St Thomas's lithotripter offered the opportunity for the first systematic evaluation of the clinical efficacy, costs, and benefits of the new technology compared with existing methods for removing stones in the United Kingdom. The Department of Health and Social Security was unable to support a randomised controlled trial of extracorporeal shock wave lithotripsy because of resistance from urologists on ethical grounds. ${ }^{1}$ Accordingly, a non-experimental comparison was performed of the clinical outcome, costs, and quality of life in patients having lithotripsy and percutaneous nephrolithotomy. This report presents the results from the first contemporaneous comparison of the clinical effectiveness of extracorporeal shock wave lithotripsy with percutaneous nephrolithotomy and allied percutaneous techniques to be undertaken in Europe. At least one similar study has taken place in the United States (J E Lingeman et al, personal communication). A comparison with open surgery was not possible because of the decline in the numbers of open operations brought about by the wide diffusion of skills in percutaneous nephrolithotomy and the advent of the St Thomas's lithotripter.

\section{Patients and methods}

Treatment by extracorporeal shock wave lithotripsy at this hospital's lithotripter centre was compared with percutaneous treatments from several hospitals in England and Scotland. All patients treated surgically for renal calculi at this hospital and participating centres were eligible for inclusion in the comparative study. Consultants were asked to notify the research team whenever a patient was treated for stones. The two groups received either percutaneous nephrolithotomy or extracorporeal shock wave lithotripsy according to the normal pattern of referrals of their general practitioners and the decisions about treatment of their consultant urologists. Patients treated by extracorporeal shock wave lithotripsy were referred by their consulting urologist or surgeon and their suitability for the treatment assessed by a panel. This is described in more detail in a previous report. ${ }^{2}$

A standard questionnaire about treatment was completed from the hospital notes of all patients who had extracorporeal shock wave lithotripsy in this hospital in the 13 months from 1 September 1985 to 30 September 1986. This group comprised NHS and private patients receiving extracorporeal shock wave lithotripsy alone or in combination with percutaneous nephrolithotomy or ureteroscopy. A similar questionnaire was completed for patients who had percutaneous nephrolithotomy either by the relevant consultant or by the research team. This group comprised patients treated by percutaneous nephrolithotomy from 1 January 1985 to 30 April 1987, with or without electrohydraulic lithotripsy, who were notified to the research team. Most of these data were collected directly from hospital notes by the research team. At two out of the three centres that provided most of the patients who had percutaneous nephrolithotomy steps were taken to obtain complete coverage of all patients who had operations for stones by searching hospital admission records. Data on the remaining patients who had percutaneous nephrolithotomy were completed by the relevant consultant urologist at the hospital of treatment.

A brief follow up questionnaire was sent to the relevant consultant at three months to assess the patient's recovery, complications, and readmissions. When the patient had not been seen by his or her consultant during these three months information was collected from the patient's general practitioner. This paper reports clinical data from the initial questionnaire on treatment and the three month follow up.

The initial questionnaire on treatment elicited data on the patient's personal characteristics and history, any abnormality of the urinary tract, details of stones, preoperative investigations, the procedure performed, the postoperative course, the postoperative procedures, and the length of stay. The three month follow up covered complications since discharge, readmissions, and procedures performed, as well as questions about whether the patient had returned to normal activities and was free of stones

\section{Results}

Rate of response and missing data-In the 13 months of the study 1001 patients were treated by extracorporeal shock wave lithotripsy. Altogether 933 had sufficient data for inclusion in the analysis; 767 were NHS patients and 166 private patients. Almost no information was found apart from the name of the patient for the remaining 68 patients. Thirteen centres initially agreed to participate in the study. In practice, 162 of the 195 patients treated by percutaneous nephrolithotomy were from only three centres (Aberdeen Royal Infirmary and Guy's and Middlesex Hospitals, London). Despite efforts to ensure the completeness of each record data were missing on some of the variables in both groups of patients.

Demographic characteristics-The distribution of age and sex was similar in the two groups: $60 \%(560 / 933)$ of the group treated with extracorporeal shock wave lithotripsy and $62 \%(121 / 195)$ of the group treated with percutaneous nephrolithotomy were male and the mean age in both groups was 49 . The range of age in patients treated with shock wave lithotripsy was 4-90 years and that for those treated by nephrolithotomy 1484 years.

Clinical features-Overall, the two groups were similar in their presenting clinical characteristics-for example, $12 \%$ of patients treated by lithotripsy had an anatomical abnormality of the urinary tract as against $14 \%$ of patients treated by nephrolithotomy and equal proportions (39\%) of the two groups had had operations for stones in the urinary tract (table I). The proportion

TABLE I-Clinical features of patients treated by extracorporeal shock wave lithotripsy and percutaneous nephrolithotomy. Values are proportions (percentages) of patients

\begin{tabular}{lcc}
\hline & $\begin{array}{c}\text { Extracorporeal shock } \\
\text { wave lithotripsy } \\
(\mathrm{n}=933)\end{array}$ & $\begin{array}{c}\text { Percutaneous } \\
\text { nephrolithotomy } \\
(\mathrm{n}=195)\end{array}$ \\
\hline $\begin{array}{l}\text { Anatomical abnormality of } \\
\text { urinary tract }\end{array}$ & $99 / 833(12)$ & $27 / 187(14)$ \\
$\begin{array}{l}\text { Previous operations for stone } \\
\text { in urinary tract }\end{array}$ & $334 / 860(39)$ & $74 / 191(39)$ \\
$\begin{array}{l}\text { Type of stone: } \\
\text { Single }\end{array}$ & $538 / 872(62)$ & $119 / 194(61)$ \\
Multiple & $222 / 872(26)$ & $57 / 194(29)$ \\
Staghorn & $112 / 872(13)$ & $18 / 194(9)$ \\
\hline
\end{tabular}

of patients with single, multiple, and staghorn stones was almost identical between the two groups. In the $62 \%$ of patients treated by lithotripsy and the $61 \%$ of patients treated by nephrolithotomy who had a single stone the size and location of the stones was compared (table II). The patients treated by nephrolithotomy had slightly smaller stones on average, but again the differences were small and not significant. A slightly higher proportion of the patients treated by nephrolithotomy had stones more than $30 \mathrm{~mm}$ in diameter $(6 \% v 2 \%)$. The position of the stone was the only 
noteworthy discrepancy between the two groups. A substantially higher proportion of the single stones in patients treated by extracorporeal shock wave lithotripsy were in the upper or middle calix.

TABLE II-Diameter and location of single stones in patients treated by extracorporeal shock wave lithotripsy and percutaneous nephrolithotomy. Values are numbers (percentages) of patients

\begin{tabular}{lcc}
\hline & $\begin{array}{c}\text { Extracorporeal shock } \\
\text { wave lithotripsy } \\
(\mathbf{n}=538)\end{array}$ & $\begin{array}{c}\text { Percutaneous } \\
\text { nephrolithotomy } \\
(\mathbf{n}=119)\end{array}$ \\
\hline Diameter (mm): & & \\
$<5$ & $32(6)$ & $4(3)$ \\
$5-20$ & $429(80)$ & $95(80)$ \\
$21-30$ & $45(8)$ & $5(4)$ \\
$>30$ & $13(2)$ & $7(6)$ \\
Unknown & $19(4)$ & $8(7)$ \\
Location: & & $3(3)$ \\
Upper calix & $62(12)$ & $7(6)$ \\
Middle calix & $75(14)$ & $38(32)$ \\
Lower calix & $160(30)$ & $40(34)$ \\
Renal pelvis & $146(27)$ & $11(9)$ \\
Pelviureteral junction & $30(6)$ & $20(17)$ \\
Ureter & $63(12)$ & \\
Unknown & $2(<1)$ & \\
\hline
\end{tabular}

Details of treatment-All patients in the group treated by nephrolithotomy received percutaneous sugery, whereas $83 \%$ (774) of the group treated by lithotripsy received extracorporeal shock wave lithotripsy alone; $11 \%$ (103) received a combined treatment of extracorporeal shock wave lithotripsy and percutaneous nephrolithotomy and $6 \%(56)$ received a combination of extracorporeal shock wave lithotripsy and ureteroscopy. In the data that follow the single and combined lithotripter treatments have been analysed as a single group for the purposes of comparison with percutaneous nephrolithotomy. Thirty six per cent (334) of the group treated by extracorporeal shock wave lithotripsy were day patients transferred from other London hospitals, to which they were returned immediately after treatment. The mean length of stay (excluding readmissions) was slightly longer for patients having percutaneous nephrolithotomy $(9.0 \mathrm{v}$ $7 \cdot 9$ days), but half $(392 / 792)$ the patients treated by extracorporeal shock wave lithotripsy stayed in hospital for four days or less compared with only a quarter (48/ 194) of the patients treated by percutaneous nephrolithotomy. The modal length of stay for extracorporeal shock wave lithotripsy was two days (range 1-64 days) compared with four days for percutaneous nephrolithotomy (range 1-46 days). The ratio of the geometric mean length of stay for nephrolithotomy ( $7 \cdot 2$ days) and shock wave lithotripsy $(5 \cdot 4$ days) was $1 \cdot 3$ (95\% confidence interval $1 \cdot 2$ to $1 \cdot 5$ ).

Mortality-No patient died in the group treated with percutaneous nephrolithotomy either during the initial stay in hospital or up to three months after treatment. Two patients died in the other group: one in hospital and one between discharge and the three month follow up of coronary thrombosis and myocardial infarction, respectively. The mortality in the two treatment groups, which had a similar distribution of age, was not significantly different.

Complications-The observed rate of postoperative complications was lower in the patients treated by percutaneous nephrolithotomy (table III). An almost total lack of obstruction from fragments was seen after percutaneous nephrolithotomy. Infections of the urinary tract were more common after percutaneous nephrolithotomy, though septicaemia was more common after extracorporeal shock wave lithotripsy. Altogether $84 \%$ (783) of the patients treated by extracorporeal shock wave lithotripsy and $84 \%$ (163) of the patients treated by percutaneous nephrolithotomy were successfully followed up at three months. Seventy eight $(10 \%)$ patients treated by lithotripsy were re- ported to have suffered obstruction from fragments during this time, whereas obstruction was not reported in any patient treated by percutaneous nephrolithotomy (table IV). Altogether 12\% (94/775) of the patients treated by lithotripsy were readmitted within three months for treatment of complications compared with only $4 \%(7 / 162)$ of those treated by nephrolithotomy.

Postoperative procedures-The incidences of postoperative procedures, including percutaneous nephrolithotomy, extracorporeal shock wave lithotripsy, ureteroscopy, and nephrostomy, were similar in both groups-for example, $8 \%$ (72/933) of the group treated by extracorporeal shock wave lithotripsy received further lithotripsy, whereas $9 \%(17 / 195)$ of the other group received further percutaneous nephrolithotomy. Altogether 12\% (109/933) of the patients treated by extracorporeal shock wave lithotripsy and 14\% (27/ 195) of those treated by percutaneous nephrolithotomy had more than one procedure postoperatively.

Outcome of treatment-Outcome was ascertained at three months. Patients were defined as free of stones if no stone was visible on radiography or, in the case of radiolucent stones, on ultrasonography or intravenous urography. All patients followed up at this hospital were assessed by intravenous urography and computed tomography at thee months. At this time the proportion of patients free of stones was $45 \%(334 / 746)$ in the group treated by extracorporeal shock wave lithotripsy and $70 \%(105 / 149)$ in that treated by percutaneous nephrolithotomy when patients with new or untreated stones were excluded ( $95 \%$ confidence interval for the difference in the proportions of patients who were free of stones $17 \%$ to $34 \%, p<0.001$ ). Most of the patients in both groups who were not free of stones had fragments rather than intact stone(s). A similar proportion of patients had unchanged or only partially disintegrated stones in both groups $(10 \%(75 / 746)$ in the group treated with extracorporeal shock wave lithotripsy and $8 \%(12 / 149)$ in that treated with percutaneous nephrolithotomy).

Single stones-An equal proportion of patients having each treatment had single non-staghorn stones (table I). The rates of being free of stones at three months in patients who had single stones and were

TABLE III-Postoperative complications while in hospital by treatment. Values are numbers (percentages) of patients ${ }^{\star}$

\begin{tabular}{lcc}
\hline Complication & $\begin{array}{c}\text { Extracorporeal shock } \\
\text { wave lithotripsy } \\
(\mathbf{n}=928)\end{array}$ & $\begin{array}{c}\text { Percutaneous } \\
\text { nephrolithotomy } \\
(\mathbf{n}=195)\end{array}$ \\
\hline Renal colic & $153(16)$ & $17(9)$ \\
Obstruction from fragments & $143(15)$ & $1(1)$ \\
Urinary tract infection & $35(4)$ & $15(8)$ \\
Loin pain & $111(12)$ & $10(5)$ \\
Vomiting & $70(8)$ & $9(5)$ \\
Fever & $47(5)$ & \\
Bruising loin & $19(2)$ & \\
Septicaemia & $9(1)$ & \\
\hline
\end{tabular}

* Minimum estimates because data were incomplete and it was not possible to distinguish non-response from lack of complications.

TABLE IV-Complications between discharge and three month follow up by treatment. Values are numbers (percentages) of patients ${ }^{\star}$

\begin{tabular}{lcc}
\hline Complication & $\begin{array}{c}\text { Extracorporeal shock } \\
\text { wave lithotripsy } \\
(\mathrm{n}=783)\end{array}$ & $\begin{array}{c}\text { Percutaneous } \\
\text { nephrolithotomy } \\
(\mathrm{n}=163)\end{array}$ \\
\hline Renal colic requiring medical & & \\
treatment & $99(13)$ & $9(6)$ \\
Obstruction from fragments & $78(10)$ & $6(4)$ \\
Urinary tract infection & $63(8)$ & $3(2)$ \\
Haematuria & $19(2)$ & $6(4)$ \\
Loin pain & $34(4)$ & \\
Vomiting & $5(1)$ & \\
\hline
\end{tabular}

*Minimum estimates because data were incomplete and it was not possible to distinguish non-response from lack of complications. 
treated with percutaneous nephrolithotomy and extracorporeal shock wave lithotripsy were, respectively, $86 \%(81 / 94)$ and $58 \%(253 / 439)$ (relative odds $=4 \cdot 6$, $95 \%$ confidence interval $2 \cdot 5$ to $8 \cdot 5$ ). For all sizes of single stones the rates of being free of stones were higher after percutaneous nephrolithotomy than extracorporeal shock wave lithotripsy, although patients with large single stones were less likely to be free of stones than those with small stones after both treatments (table V). For each of six stone locations the proportion of patients who were free of stones was lower in the group treated by extracorporeal shock wave lithotripsy (table VI). Both methods seemed to be most successful for stones originally located in the ureter and pelviureteral junction than those in other locations.

TABLE V-Proportions (percentages) of patients with single stone who were free of stones at three month follow up by size of stone and treatment

\begin{tabular}{lcc}
\hline Diameter of stone $(\mathrm{mm})$ & $\begin{array}{c}\text { Extracorporeal shock } \\
\text { wave lithotripsy }\end{array}$ & $\begin{array}{c}\text { Percutaneous } \\
\text { nephrolithotomy }\end{array}$ \\
\hline$<5$ & $16 / 25(64)$ & $4 / 4(100)$ \\
$5-20$ & $210 / 356(59)$ & $68 / 74(92)$ \\
$>20$ & $17 / 44(39)$ & $4 / 9(44)$ \\
Unknown & $10 / 14(71)$ & $5 / 7(71)$ \\
\hline Total & $253 / 439^{\star}(58)$ & $81 / 94^{\star}(86)$ \\
\hline
\end{tabular}

^Total number of patients with single stones on whom data were available.

TABLE VI-Proportions (percentages) of patients with single stones who were free of stones at three month follow up by location of stone and treatment

\begin{tabular}{lcc}
\hline Location of stone & $\begin{array}{c}\text { Extracorporeal shock } \\
\text { wave lithotripsy }\end{array}$ & $\begin{array}{c}\text { Percutaneous } \\
\text { nephrolithotomy }\end{array}$ \\
\hline Upper calix & $25 / 53(47)$ & $2 / 3(67)$ \\
Middle calix & $36 / 68(53)$ & $4 / 5(80)$ \\
Lower calix & $67 / 130(52)$ & $28 / 34(82)$ \\
Renal pelvis & $60 / 111(54)$ & $24 / 29(83)$ \\
Pelviureteral junction & $22 / 25(88)$ & $7 / 7(100)$ \\
Ureter & $43 / 52(83)$ & $16 / 16(100)$ \\
\hline Total & $253 / 439^{\star}(58)$ & $81 / 94^{\star}(86)$ \\
\hline
\end{tabular}

$\star$ Total number of patients with single stones on whom data were available.

Multiple stones-For multiple stones, as for single stones, percutaneous nephrolithotomy was again more successful than extracorporeal shock wave lithotripsy. Altogether $29 \%(55 / 187)$ of the patients with multiple calculi who were treated by lithotripsy were free of stones at three months compared with $38 \%$ (16/42) of those with such stones who were treated by nephrolithotomy. For both methods of treatment the rates of being free of stones were lower for multiple stones than for single stones of all sizes.

Staghorn stones-Roughly equal percentages of patients in each group were treated for staghorn stones (table I). At three months $62 \%(8 / 13)$ of the patients with staghorn stones who were treated by nephrolithotomy and only 15\% (14/96) of those with such stones who were treated by lithotripsy were free of stones.

Outcome adjusting for patients' age and characteristics of stones-About $60 \%$ of the patients in each group had single stones. Multiple logistic regression was used to compare the outcome of the two treatments for single stones, adjusting for differences in the patients' age, and the size and position of the stone between the two groups. The strong association between the mode of treatment (extracorporeal shock wave lithotripsy $v$ percutaneous nephrolithotomy) and outcome (being free of stones $v$ not being free of stones) observed previously remained, and the estimated relative efficacy of percutaneous nephrolithotomy against extra- corporea! shock wave lithotripsy was increased from $4 \cdot 6(95 \%$ confidence interval $2 \cdot 5$ to $8 \cdot 5)$ to $6 \cdot 6(95 \%$ confidence interval $3 \cdot 0$ to $14 \cdot 6$ ).

Readmissions-Another measure of the incidence and severity of complications between discharge and three months and of the significance of the lower rates of being free of stones in the group treated by extracorporeal shock wave lithotripsy was given by the incidence of readmission to hospital in the two groups. Altogether $19 \%(146 / 775)$ of the patients treated by extracorporeal shock wave lithotripsy had been readmitted at least once by the time of the three month follow up compared with $14 \%$ (23/162) of those treated by percutaneous nephrolithotomy. A far higher proportion of the readmissions in patients treated by extracorporeal shock wave lithotripsy were for complications rather than being planned readmissions $(64 \%(94 / 146) v 30 \%(7 / 23))$. The mean length of stay of readmissions, however, was the same in both groups ( 1.5 days). This is surprisingly low, given that half $(11 / 23)$ the patients treated by nephrolithotomy who were readmitted received further percutaneous nephrolithotomy and a third (45/146) of those treated by lithotripsy were given further extracorporeal shock wave lithotripsy and almost another third (40/146) received an endoscopic ureteric operation.

Functioning of patients-Altogether $95 \%$ (617/650) of the patients treated by extracorporeal shock wave lithotripsy and $99 \%(145 / 147)$ of those treated by percutaneous nephrolithotomy were said by their consultant to have returned to normal activities at three months. Data were, however, lacking for 133 of the $783(17 \%)$ patients treated by lithotripsy and 16 of the $163(10 \%)$ treated by nephrolithotomy. Little difference in terms of the restriction of activities recorded was found between the two groups.

\section{Discussion}

Our results are from a descriptive, comparative study rather than a randomised controlled trial. With a non-experimental design questions will always arise about the effects of selection on the groups being compared, but our data are from a contemporaneous comparison with standard methods of collecting data and the same criteria of outcome in each group. The two groups of 933 patients treated by extracorporeal shock wave lithotripsy and 195 treated by percutaneous nephrolithotomy were similar in terms of age, sex, abnormalities of the urinary tract, previous operations for stones, and the size and type of stone, but the position of the stone was different between the two groups. As data were available on only a limited number of patient variables other unknown characteristics of patients in either group may have affected the results; however, adjusting the results to take the known differences into account increased, if anything, the estimated relative efficacy of percutaneous nephrolithotomy for single stones.

The different clinical teams in our study may have varied in their judgment of the outcomes of their treatments. Standard forms were, however, used throughout and variables were defined in the same way for each group. Furthermore, the definition of successful treatment was specifically chosen to be as unequivocal as possible. In the event, no significant differences were found in either presenting characteristics or outcome between the $17 \%(33 / 195)$ of patients treated by percutaneous nephrolithotomy who did not come from the three main centres (Aberdeen and Guy's and Middlesex Hospitals) and the other patients in the group treated by percutaneous nephrolithotomy. Furthermore, no significant differences were found in the rates of being free of stones in patients with single stones among the three main 
centres, with and without adjusting for age and size and position of stone $(0 \cdot 1<\mathrm{p}<0 \cdot 25)$. These results should, however, be treated with caution because of the small numbers.

The first results of the use of extracorporeal shock wave lithotripsy in the United Kingdom were encouraging. Wickham et al treated the first 50 patients and reported that the procedure was safe and effective, with a short stay in hospital and an extremely brief convalescence, and concluded that extracorporeal shock wave lithotripsy was better than all other methods of removing renal stones. ${ }^{3}$ Subsequently, Charig et al published the results from 1052 consecutive patients with renal calculi who were treated by open surgery (from 1972 to 1980), percutaneous nephrolithotomy (from 1980 to 1985), and extracorporeal shock wave lithotripsy (in 1985). ${ }^{+}$Their study was based on historical controls and they used different criteria to judge the success of the different treatments. They concluded that extracorporeal shock wave lithotripsy was better than both open surgery and percutaneous nephrolithotomy for most types of stone except cystine and staghorn calculi. Ninety per cent of the patients treated by extracorporeal shock wave lithotripsy and a combination of lithotripsy and percutaneous nephrolithotomy were described as successfully treated at three months as against $83 \%$ of the patients treated by percutaneous nephrolithotomy; $24 \%$ of 350 patients treated by lithotripsy had "gravel" but no adverse clinical effects. The rates of success of the three modes of treatment for all sizes of stones were not, however, significantly different and no information was given on potentially important variables such as the position of the stone. The three groups of patients had stones which were dissimilar in size. Furthermore, for percutaneous nephrolithotomy success was defined as being free of stones at three months, as in our study, whereas for extracorporeal shock wave lithotripsy success was equated with the fragmentation of stones into particles less than $2 \mathrm{~mm}$ in diameter.

Although the proportion of subjects who were free of stones after treatment was the traditional yardstick of success of treatments for renal stones before extracorporeal shock wave lithotripsy, many of the more impressive reports of success with extracorporeal shock wave lithotripsy have been based on less stringent criteria. ${ }^{3.11}$ Until more is known of the long term consequences and rates of recurrence associated with retained small fragments it is premature to assume that such fragments are benign. Thus, the rate of being free of stones should be used as the appropriate measure of success.

Three other studies of extracorporeal shock wave lithotripsy and percutaneous nephrolithotomy gave rates of being free of stones. In all three comparison with our study has difficulties in the comparability of the stones treated and the number of patients lost to follow up. In a Swedish study currently continuing at Linkoping $49 \%$ of patients treated by extracorporeal shock wave lithotripsy were free of stones at four weeks (B Jonsson, joint EHPF and World Health Organisation meeting, Brussels, 1987), compared with $45 \%$ of such patients in our study. This suggests a better outcome in Sweden as, apart from the proportion of staghorn stones, the stones were on average larger than those in our study. In two American studies the rates of being free of stones were higher than those from either Linkoping or St Thomas's. Riehle et al from New York defined $75 \%$ of "treatments" as getting rid of stones at three months. ${ }^{12}$ Unfortunately, it is not possible to ascertain whether the patients treated were comparable with ours. In addition, stents were used, which was not the case in our study. Lingeman et al found similar results in a series of 982 patients from Indianapolis, with $72 \%$ of patients being free of stones at three months. ${ }^{13}$ Only $4 \%$ of patients returning for follow up at three months had clinically important residual fragments or required operations to remove calculi. This study was similar to ours in terms of the size of the stones but not in terms of the position of the stones, which is known to affect outcome significantly. Unpublished worldwide results reported at the fourth symposium on shock wave lithotripsy held in Indianapolis, United States, in March 1988 suggested that the rates of being free of stones were $60-70 \%$ in unselected patients.

Comparisons of the effectiveness of new technology are often complicated by the rapid evolution of techniques and the development of surgical skills. In our study, however, the difference between the outcomes of the two treatments does not seem to be accounted for, even in part, by a process of learning how best to use the new techniques of extracorporeal shock wave lithotripsy. We did not begin to collect data on patients treated by extracorporeal shock wave lithotripsy until the surgical team had six months' experience with the new technology, and no significant trend in outcome (being free of stones $v$ not being free of stones) was found over the 13 months of the study. Since the study was completed stents have been introduced to ease the clearance of fragments from the ureter and this may have improved results. As yet, however, no evidence exists that stents reduce the rate of retreatment or the ultimate results of treatment. Adjuvant techniques may have improved, but techniques of percutaneous nephrolithotomy have also developed. ${ }^{14}$ Our results for extracorporeal shock wave lithotripsy are for the practice at one hospital. Higher rates of being stone free might have been obtained, at an increased cost, by treating each patient more intensively.

The data on length of stay for the two methods of treatment are not easy to interpret. The mean length of stay for lithotripsy was high, 7.9 days, but the mode was only two days. This suggests that many patients were staying only a short time for their treatment. A few patients who stayed a long time raised the mean, but the reasons for these long stays were not recorded.

The long term effects of extracorporeal shock wave lithotripsy are unknown. The consequences for recurrence of stones of the high prevalence of debris in the group treated by extracorporeal shock wave lithotripsy (37\% at three months) need to be carefully monitored. This is a worrying feature of extracorporeal shock wave lithotripsy as it is generally accepted that residual fragments after open surgery or percutaneous nephrolithotomy result in a greatly increased rate of recurrence requiring further treatment. ${ }^{15}$ Some evidence of short term effects on renal function exists, ${ }^{5}{ }^{16-18}$ and the possibility of long term adverse consequences cannot be ruled out. Hypertension is also a possibility. As part of the current study clinical data are being collected on patients treated by shock wave lithotripsy at six, 12, and 24 months after discharge. Data on the costs of treatment and on patients' experiences of treatment, which are required to evaluate the two treatments fully, have been collected and will be reported separately.

\section{CONCLUSIONS}

Despite the lack of published evidence of the comparative merits of percutaneous nephrolithotomy and extracorporeal shock wave lithotripsy in which differences among patients and stones have been taken into account, the Scottish National Specialist Services Advisory Committee recommended in 1987 that a national lithotripsy service should be set up for Scotland, on the grounds that extracorporeal shock wave lithotripsy was $90 \%$ effective and less stressful for the patient than alternative methods of treatment. ${ }^{19}$ 
Plans are already well advanced to install new generation lithotripters for treating renal stones in a number of NHS regions. Lithotripsy is currently being brought into clinical practice for the destruction of gall stones. Yet the more extravagant claims made for extracorporeal shock wave lithotripsy were not upheld in our study of routine practice. The clinical results of this first contemporaneous comparison of extracorporeal shock wave lithotripsy and percutaneous nephrolithotomy in Europe raise doubts about the superiority of extracorporeal shock wave lithotripsy over alternative techniques for treating renal calculi in two groups of patients with similar demographic and stone characteristics.

We thank Linda Jarrett, Meredith Temple-Smith, and Margot Halse, the former research assistants on the project, for their hard work in collecting much of the data, and Ann Petruckevitch for analysing the data in the late stages of preparing the manuscript. We thank the staff of the St Thomas's Lithotripter Centre and of the departments of urology at Guy's and Middlesex Hospitals, London, for access to patients' records, and $\mathrm{Mr} \mathrm{J}$ Steyn and his secretary, Mrs Nealon, for providing data on patients treated at the Aberdeen Royal Infirmary. We also thank Messrs K E D Shuttleworth and M I Bultitude, consultant urologists at St Thomas's, for their advice during the design of the study and for their helpful comments on the manuscript, and two anonymous referees for constructive criticism. We gratefully acknowledge the financial support of the Department of Health and Social Security in undertaking the study.

1 Challah S, Mays N. The randomised controlled trial in the evaluation of new technology: a case study. Br Med I 1986;292:877-9.

2 Palfrey ELH, Bultitude MI, Challah S, Pemberton J, Shuttleworth KED
Report on the first 1000 patients treated at St Thomas' Hospital by extracorporeal shock wave lithotripsy. Br 7 Urol 1986;58:573-7.

3 Wickham J, Webb DR, Payne SR, Kellett MJ, Watkinson G, Whitfield HN Extracorporeal shock wave lithotripsy: the first 50 patients treated in Britain. Br Med J 1985;290:188-9.

4 Charig CR, Webb DR, Payne SR, Wickham JEA. Comparison of treatment of renal calculi by open surgery, percutaneous nephrolithotomy, and extracorporeal shock wave lithotripsy. Br Med J 1986;292:879-82.

5 Chaussy C, Schmiedt E, Jocham D, Schuller J, Brandl H, Liedl B Extracorporeal shock-wave lithotripsy (ESWL) for treatment of urolithiasis. Urology 1984;23 (suppl):59-66.

6 Blume E. Sound, shock waves shatter kidney stones. FAMA 1983;249:2434-5. Chaussy C, Schmiedt E. Shock wave treatment for stones in the upper urinary tract. Urol Clin North Am 1983;10:743-50.

8 Chaussy C, Schmiedt E. Extracorporeal shock wave lithotripsy (ESWL) for kidney stones: an alternative to surgery? Urol Radiol 1984;6:80-7.

9 Fuchs G, Miller K, Rassweiler J, Eisenberger F. Alternatives to open surgery for renal calculi: percutaneous nephrolithotomy and extracorporeal shockwave lithotripsy. Klinische und Experimentelle Urologie 1984;8:153-77.

10 Miller K, Fuchs G, Rassweiler J, Eisenberger F. Financial analysis, personnel planning and organizational requirements for the installation of a kidney planning and organizational requirements for the installation
lithotripter in a urologic department. Eur Urol 1984;10:217-21.

11 Das G, Dick J, Bailey MJ, et al. Extracorporeal shock wave lithotripsy: first 1000 cases at the London Stone Clinic. Br Med J 1987;295:891-3.

12 Riehle RA, Fair WR, Vaughan ED. Extracorporeal shock-wave lithotripsy for upper urinary tract calculi: one year's experience at a single center. $尹 A M A$ 1986;255:2043-8.

13 Lingeman JE, Newman D, Mertz JHO, el al. Extracorporeal shock-wave lithotripsy: the Methodist Hospital of Indiana experience. $\mathcal{O}$ Urol 1986;135: 1134-7.

14 Lee WJ, Smith AD, Cubelli V, Vernace FM. Percutaneous nephrolithotomy: analysis of 500 consecutive cases. Urol Radiol 1986;8:61-6.

15 Sleight $W$, Wickham JEA. Long term follow up of 100 cases of renal calculi. Br J Urol 1977;49:601-4.

16 Kaude JV, Williams CM, Millner MR, Scott KN, Finlayson B. Renal morphology and function immediately after extracorporeal shock-wave morphology and function immediately after extracorpore

17 Baumgartner BR, Dickey KW, Ambrose SS, Walton KN, Nelson RC, Bernardino ME. Kidney changes after extracorporeal shock-wave lithotripsy: appearance on MR imaging. Radiology 1987;163:531-4

18 Newman RC, Bezirdjian L, Steinbock G, Finlayson B. Complications of extracorporeal shock wave lithotripsy: prevention and treatment. Semin Urol 1986;4:170-4

19 National Specialist Services Advisory Committee (NSSAC). Lithotripsy. Health Bull (Edinb) 1987;45:2.

(Accepted 28 April 1988)

\title{
Cigarette smoking as risk factor for late fetal and early neonatal death
}

\author{
Sven Cnattingius, Bengt Haglund, Olav Meirik
}

\section{Uppsala University Hospital, S-751 85 Uppsala, Sweden Sven Cnattingius, $\mathrm{MD}$, associate professor in social medicine \\ Bengt Haglund, DRMEDSC, research associate, centre for} primary care research

Human Reproduction Programme, World Health Organisation, Geneva, Switzerland

Olav Meirik, MD, medical officer

Correspondence to: Dr Cnattingius.

\begin{abstract}
Risk factors for late fetal death and early neonatal mortality were examined in a population based prospective study. Practically all Swedish births between 1983 and 1985 were included, 281808 births in all. The overall rates of late fetal death and early neonatal mortality were 3.5 and 3.1 per 1000 , respectively. About $30 \%$ of the pregnant women were recorded as being daily smokers. Logistic regression analyses showed significant relative risks for late fetal death for high maternal age (1.4), nulliparity $(1 \cdot 4)$, multiparity $(\geqslant 2)(1 \cdot 3)$, smoking $(1 \cdot 4)$, and multiple births $(2 \cdot 8)$. Significant relative risks for early neonatal mortality were found for multiple births (4.9) and smoking (1.2). Smokers aged under 35 faced a relative risk of late fetal death ranging from 1.1 to 1.6 , while the risk for late fetal death was doubled i' the mothers were aged 35 years or more and smoked.
\end{abstract}

In countries like Sweden, where maternal cigarette smoking is prevalent, smoking may be the most important preventable risk factor for late fetal death.

\section{Introduction}

The causal association between maternal cigarette smoking and low birth weight is generally accepted, whereas the association between smoking and perinatal death is disputed.' In a review article McIntosh has reported that only five of 17 studies have found significantly increased risks of stillbirth among smokers. ${ }^{2}$ An increased risk of early neonatal death among the infants of smokers was reported in four studies, though none reached significance. The results of a large study of more than 360000 single births in Missouri, United States, have also recently been published. ${ }^{3}$ In the multivariate analyses performed a significant effect of smoking on late fetal death and neonatal mortality (0-28 days) was seen. It has further been suggested that the risk of perinatal death related to smoking may not be equally distributed: it has been reported to vary with such factors as maternal age, parity, ethnic group, and socioeconomic state. ${ }^{+}$ The above studies, including the Missouri study, were based on retrospectively collected data from fairly heterogeneous populations that had perinatal mortality rates ranging from 12 to 30 per 1000 .

In Sweden the overall perinatal mortality is below seven per 1000 . The population is homogeneous, with poverty and undernutrition practically non-existent in the pregnant population. Antenatal and obstetric care is free, and practically all pregnant women visit the antenatal clinics regularly during pregnancy and give birth at hospitals. 'Thus Sweden provides an excellent opportunity to study late fetal death and early neonatal mortality resulting from biological variation and specific environmental influences in a well nourished population.

In 1973 a medical birth registry was set up by the National Board of Health and Welfare in Sweden. ${ }^{6}$ Since 1982 information has been prospectively collected for each pregnancy from the first antenatal visit. These 